\title{
Single- and Two-Photon Fluorescence Recovery after Photobleaching
}

\author{
Kelley D. Sullivan, Ania K. Majewska, and Edward B. Brown
}

\begin{abstract}
Fluorescence recovery after photobleaching (FRAP) is a microscopy technique for measuring the kinetics of fluorescently labeled molecules and can be applied both in vitro and in vivo for two- and three-dimensional systems. This introduction discusses the three basic FRAP methods: traditional FRAP, multiphoton FRAP (MPFRAP), and FRAP with spatial Fourier analysis (SFA-FRAP). Each discussion is accompanied by a description of the mathematical analysis appropriate for situations in which the recovery kinetics is dictated by free diffusion. In some experiments, the recovery kinetics is dictated by the boundary conditions of the system, and FRAP is then used to quantify the connectivity of various compartments. Because the appropriate mathematical analysis is independent of the bleaching method, the analysis of compartmental connectivity is discussed last, in a separate section.
\end{abstract}

Since its introduction in the 1970s (Peters et al. 1974; Axelrod et al. 1976), fluorescence recovery after photobleaching (FRAP) has been used to measure the diffusion coefficient (or analogous transport parameters) of labeled molecules in both two-dimensional systems such as cell membranes, small regions of cells, and lamellipodia (Feder et al. 1996; Braga et al. 2007) and three-dimensional systems such as tumor tissues or cell bodies (Chary and Jain 1989; Berk et al. 1997; Pluen et al. 2001; Stroh et al. 2004; Chauhan et al. 2009). Each of the three FRAP techniques is performed by first photobleaching a small region of interest within a sample, and then monitoring the region as fluorescent molecules from outside the region diffuse in to replace the photobleached molecules. The original spot FRAP technique has undergone a variety of modifications to accommodate different photobleaching methods, including patterned (Abney et al. 1992), continuous (Wedekind et al. 1996), line (Braeckmans et al. 2007), and disk-shaped (Mazza et al. 2008) photobleaching. Modifications to the recovery analysis have also expanded FRAP as a tool to analyze binding kinetics (Kaufmann and Jain 1991; Berk et al. 1997; Schulmeister et al. 2008), to quantify the connectivity of compartments (Majewska et al. 2000; Cardarelli et al. 2007), and to investigate polymer structure-property relationships (Li et al. 2010).

In an FRAP experiment, a focused laser beam bleaches a region of fluorescently labeled molecules in a thin sample of tissue (Axelrod et al. 1976). The same laser beam, greatly attenuated, then generates a fluorescence signal from that region as unbleached fluorophores diffuse in. A photomultiplier tube, or a similar detector, records the recovery in fluorescence signal, producing a fluorescence-versus-time

Adapted from Imaging: A Laboratory Manual (ed. Yuste). CSHL Press, Cold Spring Harbor, NY, USA, 2011.

(C) 2015 Cold Spring Harbor Laboratory Press

Cite this introduction as Cold Spring Harb Protoc; doi:10.1101/pdb.top083519 
K.D. Sullivan et al.

curve. In a conventional (one-photon) FRAP experiment, simple analytical formulas can be fit to the fluorescence recovery curve to generate the two-dimensional diffusion coefficient of the fluorescent molecule but only if the sample is sufficiently thin (see FRAP Diffusion Analysis). If the sample is not thin enough for the analytical solution to hold, the diffusion coefficient can be estimated by comparing the recovery time with that of molecules with known diffusion coefficients in samples of identical thickness.

In an MPFRAP experiment, a focused beam from a mode-locked laser provides both bleaching and monitoring, generating fluorescence and photobleaching via multiphoton excitation (Brown et al. 1999). Most current MPFRAP experiments are designed for two-photon fluorescence and photobleaching; however, single- and multiple (three or higher)-photon excitation can also be achieved. Therefore, in this introduction, we will refer to these experiments generally as "multiphoton." The intrinsic spatial confinement of multiphoton excitation means that the bleaching/monitoring volume is three-dimensionally resolved (Denk et al. 1990); consequently, there is no upper limit on the sample thickness. Simple analytical formulas can be applied to the fluorescence recovery curve to generate the three-dimensional diffusion coefficient of the fluorescent molecule.

\section{FRAP Instrumentation}

The primary instrumentation of one-photon FRAP consists of a laser source, an acousto-optic modulator (AOM), a dichroic mirror, an objective lens, a gated photomultiplier tube (PMT), and a data recording system such as an analog-to-digital (A/D) board or scaler (photon-counting device) (Fig. 1A). The laser source is directed through the AOM to the dichroic mirror and the objective lens and into the fluorescent sample.

The laser is typically an argon-ion laser operating in the lowest-order transverse electromagnetic $\left(\mathrm{TEM}_{00}\right)$ mode to produce a Gaussian transverse intensity profile, suitable for analysis of recovery curves (see FRAP Diffusion Analysis). The laser must be modulated on a much faster timescale than the diffusive recovery time of the system, often requiring modulation times of fractions of a millisecond. This necessitates the use of an AOM as the beam-modulation device because of its fast response time. To generate significant variation in transmitted intensity, the first diffraction maximum of the AOM should be used, not the primary transmitted beam.

\section{MPFRAP Instrumentation}

The primary instrumentation of MPFRAP consists of a laser source, a Pockels cell, a beam expander, a dichroic mirror, an objective lens, a gated PMT, and a data recording system (Fig. 1B). The laser source is directed through the Pockels cell to the beam expander, the dichroic mirror, and the objective lens and into the fluorescent sample.

The laser is typically a mode-locked (100-fsec pulses) Ti:sapphire laser. This beam is expanded to overfill the objective lens (Zipfel et al. 2003), thereby producing a uniformly illuminated back aperture, resulting in the formation of the highest resolution spot in the plane of the sample (Pawley 2006). The intrinsic spatial confinement of multiphoton excitation produces a three-dimensionally defined
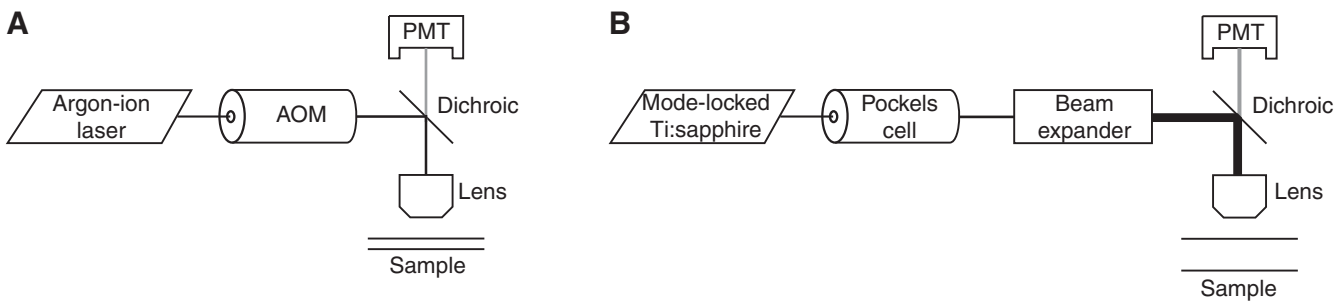

FIGURE 1. (A) Equipment for fluorescence recovery after photobleaching (FRAP). (B) Equipment for multiphoton FRAP (MPFRAP). Laser light is passed through the AOM or the Pockels cell/beam expander and directed by the dichroic to the objective lens, which focuses the light within the sample. Fluorescence from the sample then passes back through the lens and the dichroic to the PMT. 
bleach volume, whose size depends on the numerical aperture (NA) and the wavelength of the excitation light and is typically $\sim 0.5 \times 0.5 \times 1 \mu \mathrm{m}$. This extremely small bleached volume dissipates rapidly (hundreds of microseconds for smaller fluorescently labeled molecules such as fluorescein isothiocyanate [FITC]-bovine serum albumin [BSA] or green fluorescent protein [GFP]). Consequently, MPFRAP requires a beam-modulation system with response times as fast as $1 \mu$ sec. The AOM traditionally used in one-photon FRAP relies on diffraction of the laser beam to achieve intensity modulation, whereas 100-fsec pulses, typical of a mode-locked Ti:sapphire laser used in multiphoton FRAP, have a bandwidth of $15 \mathrm{~nm}$. Different wavelengths of light will diffract in different directions; therefore, the nondiffractive Pockels cell is often used for MPFRAP beam modulation instead of an AOM. A Pockels cell operates by passing a beam through a crystal, across which a voltage is applied. Varying the voltage rotates the plane of polarization of the incident light. Before exiting the Pockels cell, the beam passes through a polarizer, which converts the rotation in the plane of polarization to a variation in intensity.

\section{FRAP or MPFRAP Procedure}

1. Set the laser modulator (AOM or Pockels cell) to a low transmission state, producing the monitor beam and generating fluorescence from the sample, which is collected by the objective lens and is detected by the PMT. The scaler monitors the output of the PMT for a short duration (tens of microseconds to milliseconds), recording the prebleach signal.

2. Switch the laser power modulator to a high transmission level, producing the bleach beam, which photobleaches a fraction of the fluorophores within the sample. The modulator is returned rapidly to the low monitoring state (after a total bleach time that depends on the sample dynamics). If possible, gate the PMT during the bleach pulse to avoid damage to the PMT.

3. Continuously record the fluorescence generated by the monitor beam. As unbleached fluorophores diffuse into the region excited by the laser beam, the fluorescence signal recovers to equilibrium levels.

4. Analyze the fluorescence recovery curve to yield diffusion coefficients or analogous parameters as well as the fraction of immobile fluorophores (Fig. 2).

\section{Considerations when Determining FRAP and MPFRAP Diffusion Parameters}

A number of steps must be taken to ensure accurate determination of the diffusion parameters.

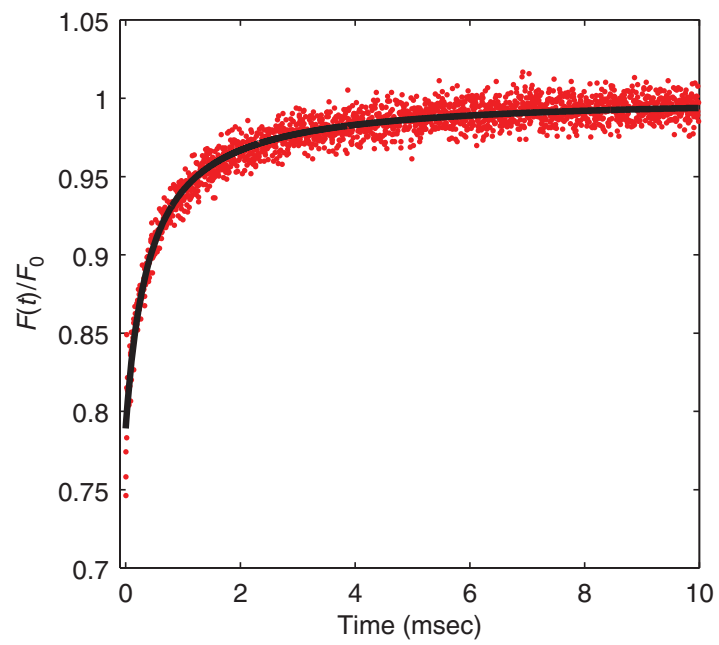

FIGURE 2. MPFRAP recovery curve. MPFRAP was performed on FITC-BSA in free solution. (Solid line) Least- $\chi^{2}$ fit, producing a diffusion coefficient at $22^{\circ} \mathrm{C}$ of $52.2 \mu \mathrm{m}^{2}$ / sec. 
1. The power of the monitoring beam must be high enough to generate a sufficient fluorescence signal but not so high as to cause significant photobleaching. The generation of photobleaching by the monitoring beam can be detected easily by photon counting over an integration time much larger than the expected recovery time for a range of typical monitoring powers. For MPFRAP with two-photon excitation, a reduction from a slope of 2 on a log-log plot of photon counts as a function of power indicates the presence of photobleaching. For FRAP, a deviation from a slope of 1 on a linear plot of photon counts as a function of power indicates the presence of photobleaching. This is because fluorescence scales as intensity to the $n$th power, where $n$ is the number of photons absorbed during excitation. In a one-photon FRAP experiment, if the resultant monitoring power is too low to allow a sufficient signal-to-noise ratio, the monitoring beam can be repeatedly cycled between 0 and a high power that causes some limited photobleaching, allowing intermittent recording of the fluorescence recovery at higher signal rates, while limiting the total photobleaching by the monitoring beam (Waharte et al. 2005).

2. The duration of the bleaching flash must be short enough that no significant diffusion (i.e., recovery in the fluorescent signal) occurs during the bleach pulse. A rule of thumb is that the bleach pulse should be less than one-tenth of the half-recovery time of the subsequent recovery curve.

3. If the acquisition of the fluorescence recovery curve does not occur for a long enough period, overestimation of the immobile fraction and underestimation of the diffusion recovery time can result. A rule of thumb is that the recovery curve should be visibly flat (i.e., any systematic change in signal is less than shot noise) for the latter half of the recording time.

4a. In FRAP and MPFRAP, the fluorescence excitation rate is assumed to scale as rate $\sim \sigma\left\langle I^{b}\right\rangle$, where $\sigma$ is the absorption cross section (units of $\mathrm{cm}^{2}$ for one-photon excitation and $\mathrm{cm}^{4} \mathrm{sec}$ for two-photon excitation), $I$ is the intensity of the bleach beam, $b$ is the number of photons absorbed in a bleaching event, and $<>$ denotes a time average. There is an upper limit to the excitation rate of a fluorescent molecule, however, because fluorescent molecules have excited-state lifetimes of $t_{\mathrm{L}} \approx 10 \mathrm{nsec}$ and, hence, cannot be excited at a faster rate than $1 / t_{\mathrm{L}}$. Furthermore, the pulsed lasers used in MPFRAP have a duty cycle of $t_{\mathrm{D}}=12.5 \mathrm{nsec}$. Consequently, when the excitation rate of a fluorophores during the bleaching pulse approaches a significant fraction of $1 / t_{\mathrm{L}}$ or $1 / t_{\mathrm{D}}$, the rate of excitation will deviate from $\sim \sigma<I^{b}>$ and will asymptotically approach a limiting value, which depends on $1 / t_{\mathrm{L}}$ or $1 / t_{\mathrm{D}}$. This phenomenon is known as excitation saturation. FRAP or MPFRAP curves generated in the saturation regime in which the photobleaching rate does not scale as $\sim \sigma<I^{b}>$, will produce erroneously low diffusion coefficients.

4b. To avoid excitation saturation, a series of FRAP or MPFRAP curves must be generated at increasing bleach powers. The curves are then analyzed (see the section on Diffusion Analysis) using the bleach depth parameter and the diffusive recovery time as the fitting parameters. For MPFRAP with two-photon excitation, a reduction from a slope of 2 on a log-log plot of bleach depth parameter as a function of power indicates the presence of excitation saturation. For FRAP, a deviation from a slope of 1 on a linear plot of bleach depth parameter as a function of power indicates the presence of excitation saturation.

5. To convert a measured diffusive recovery time to a diffusion coefficient, the characteristic size of the bleached region must be known. In both FRAP and MPFRAP, the excitation probability as a function of position transverse to the beam axis at the focal spot (i.e., the transverse beam intensity profile to the $n$th power) can be well represented by a Gaussian function (see below), whose characteristic half-width at $e^{-2}$ must, therefore, be determined to convert recovery times to diffusion coefficients. In the case of one-photon FRAP, the $e^{-2}$ half-width of the excitation probability must be measured transverse to the beam axis only, whereas in MPFRAP, the $e^{-2}$ half-width in both the transverse and the axial directions must be measured. This is typically accomplished by scanning the focus of the laser beam across a subresolution ( $\sim 10 \mathrm{~nm}$ or less) fluorescent bead and recording the fluorescent signal versus the position of the bead. Unless the 
excitation beam of a one-photon FRAP system is provided by a confocal laser-scanning microscope, there is no mechanism for easily altering the position of the laser focus in the sample plane, so a simple method to measure the excitation probability is to scan a subresolution bead transversely across the stationary beam focus with a stepper motor or a piezoelectric motor (Schneider and Webb 1981). In an MPFRAP system, the laser position is usually governed by galvanometers and stepper motors as part of a multiphoton laser-scanning microscope system. Consequently, it is relatively easy to scan the laser across a stationary subresolution fluorescent bead to determine the transverse $e^{-2}$ half-width, whereas the axial $e^{-2}$ half-width can be determined by scanning the bead across the focus, using the focus stepper motor that accompanies most laser-scanning microscope systems.

\section{Diffusion Analysis: Conventional (One-Photon) FRAP}

If the sample thickness in an FRAP experiment is sufficiently thin, the complex three-dimensional hourglass shape of the focused bleaching beam (Pawley 2006) can be ignored. This is because bleaching only occurs in a thin slice at the focus of the beam, and the postbleach recovery kinetics occurs laterally in a two-dimensional system. If the excitation laser is operating in the $\mathrm{TEM}_{00}$ mode and significantly underfills the objective lens (i.e., the beam is significantly smaller than the back aperture of the objective lens), then the transverse intensity profile is a simple Gaussian one, and an analytical formula for the fluorescence recovery curve can be derived. For a Gaussian laser beam, the fluorescence recovery curve describing free diffusion in a two-dimensional system is given by Axelrod et al. (1976).

$$
F(t)=F_{\infty} \sum_{n=0}^{\infty} \frac{(-\beta)^{n}}{n !} \frac{1}{\left(1+n+2 n t / \tau_{\mathrm{D}}\right)},
$$

where $F_{\infty}$ is the $t=\infty$ fluorescence signal, $\beta$ is the bleach depth parameter, and $\tau_{\mathrm{D}}$ is the twodimensional diffusion recovery time. The fraction of immobile fluorophores in the sample is given by $\left(F_{0}-F_{\infty}\right) / F_{\infty}$, where $F_{0}$ is the prebleach fluorescence signal and immobile is defined as having a diffusive mobility significantly slower than the timescale of the experiment. The diffusion coefficient is given by $D=w^{2} / 4 \tau_{\mathrm{D}}$, where $w$ is the transverse $e^{-2}$ half-width of the laser beam at the sample.

FRAP can be extended to thicker samples, but analytic derivations of the diffusion coefficient become problematic because of the complex nature of the hourglass-shaped laser focus distribution. Furthermore, the fluorescence recovery time becomes dependent on the thickness of the sample, which may or may not be known. In these cases, the FRAP technique is often limited to a simple comparison of recovery times between samples of unknown diffusion coefficients and samples with known diffusion coefficients that have been measured with analytical techniques such as those described above.

\section{Diffusion Analysis: MPFRAP}

In an MPFRAP experiment, the highest spatial resolution is achieved by overfilling the objective lens, producing a diffraction-limited intensity distribution at the beam focus. The square (or higher power) of this intensity profile is well approximated by a Gaussian distribution, both transverse to and along the optical axis, although the half-width at $e^{-2}$ is typically longer in the axial dimension than in the transverse dimension (Pawley 2006). For an overfilled objective lens inducing two-photon fluorescence and photobleaching, the fluorescence recovery curve describing free diffusion in a three-dimensional system is given by Brown et al. (1999) as

$$
F(t)=F_{\infty} \sum_{n=0}^{\infty} \frac{(-\beta)^{n}}{n !} \frac{1}{\left(1+n+2 n t / \tau_{\mathrm{D}}\right)} \frac{1}{\sqrt{1+n+2 n t /\left(R \tau_{\mathrm{D}}\right)}},
$$


K.D. Sullivan et al.

where $F_{\infty}$ is the $t=\infty$ fluorescence signal, $\beta$ is the bleach depth parameter, and $\tau_{\mathrm{D}}$ is the threedimensional diffusion recovery time. Because the signal is limited by restrictions on the bleaching and the monitoring powers (as described in the section, Considerations when Determining FRAP and MPFRAP Diffusion Parameters, above), MPFRAP experiments are generally performed with a series of bleach/monitor sequences at a location of interest, and the resultant curves averaged together to improve the signal-to-noise ratio. Therefore, MPFRAP is insensitive to immobile fluorophores, as they are bleached out during repeated flashes and do not contribute to recovery kinetics. The diffusion coefficient is given by $D=w_{r}^{2} / 8 \tau_{\mathrm{D}}$, where $w_{r}$ is the transverse $e^{-2}$ half-width of the laser beam at the sample and $R$ is the square of the ratio of the axial $\left(w_{z}\right)$ to the radial $\left(w_{r}\right) e^{-2}$ halfwidth (Fig. 2).

In some experiments, it may be the case that the mobility of the fluorophore of interest is influenced by convective flow as well as diffusion. In this case, the shape of the fluorescence recovery curve changes and so must the mathematical model describing the recovery. By introducing a coordinate shift into the derivation of the equation immediately above, the new derivation yields (Sullivan et al. 2009)

$$
F(t)=F_{0} \sum_{n=0}^{\infty} \frac{(-\beta)^{n}}{n !} \frac{\exp \left[\frac{4 n t\left(1 / \tau_{v_{x}}^{2}+1 / \tau_{v_{y}}^{2}\right)}{1+n+2 n t / \tau_{\mathrm{D}}}\right] \exp \left[\frac{4 n t / \tau_{v_{z}}^{2}}{1+n+2 n t / R \tau_{\mathrm{D}}}\right]}{\left(1+n+2 n t / \tau_{\mathrm{D}}\right)\left(1+n+2 n t / R \tau_{\mathrm{D}}\right)^{1 / 2}},
$$

where $\tau_{v x}=w_{r} / v_{x}, \tau_{v y}=w_{r} / v_{y}$, and $\tau_{v z}=w_{z} / v_{z}$; and $v^{2}=v_{x}^{2}+v_{y}^{2}+v_{z}^{2}$ is the speed of the convective flow. All other variables are defined as above. With this diffusion-convection model, accurate values for the diffusion coefficient can be obtained even under the influence of moderate flows, defined by a dimensionless speed parameter, $v_{s}=v\left(w_{r} / 8 D\right) \leq 3$ (Fig. 3) (Sullivan et al. 2009).

In an SFA-FRAP experiment, a focused laser beam is used to bleach a region of fluorophore, as in FRAP (described above). Unlike conventional FRAP, however, the evolution of the bleached region is
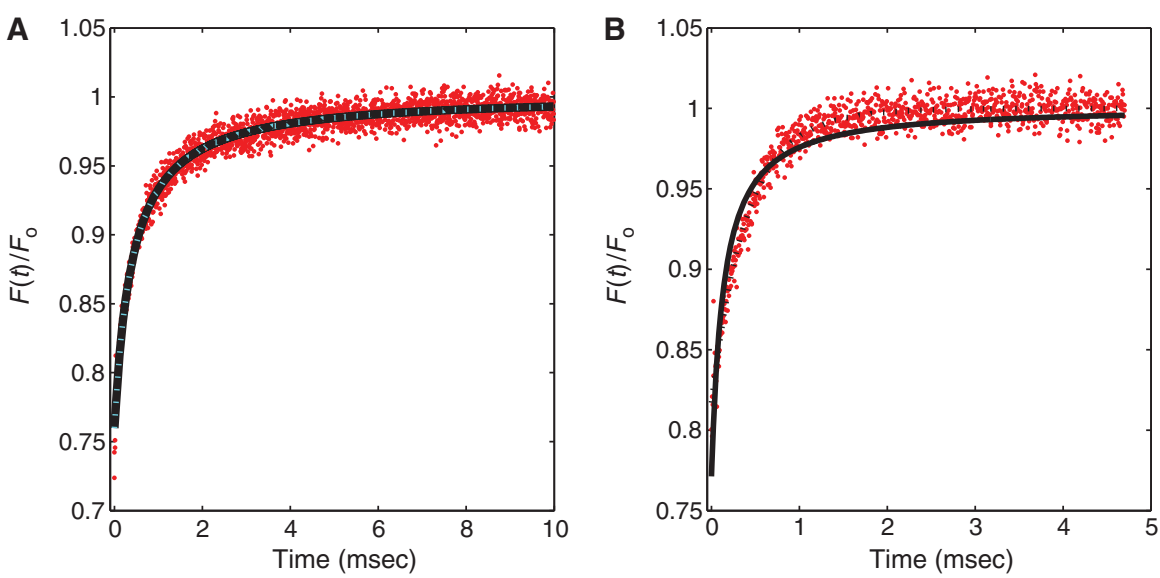

FIGURE 3. MPFRAP recovery curves. In both $A$ and $B$, MPFRAP was performed on FITC-BSA in free solution, and the data were fit to both the conventional diffusion-only (solid line) and the diffusion-convection (dashed line) models. $(A)$ Solution shows no convective flow. Both fits yield a diffusion coefficient at $22^{\circ} \mathrm{C}$ of $52.9 \mu \mathrm{m}^{2} / \mathrm{sec}$. (B) Solution shows convective flow with a scaled speed of 0.8. The diffusion-only model fits the data poorly and yields a diffusion coefficient at $22^{\circ} \mathrm{C}$ of $160 \mu \mathrm{m}^{2} / \mathrm{sec}$. The diffusion-convection model fits the data well and yields a diffusion coefficient of $51.9 \mu \mathrm{m}^{2} / \mathrm{sec}$, in good agreement with the value determined in the absence of convective flow. 
imaged repeatedly using a charge-coupled device (CCD) camera with wide-field illumination provided by a mercury lamp. The sequential images of the recovery of the bleached spot are Fourier transformed, and the decay of selected spatial frequency components produces the diffusion coefficient of the diffusing fluorophore, without requiring knowledge of the details of the bleaching distribution or the sample thickness (Tsay and Jacobson 1991; Berk et al. 1993). Consequently, SFA-FRAP can be performed in thick samples, although its reliance on epifluorescence means that the diffusion coefficients it measures are averages over the entire depth of view of the microscope and are not three-dimensionally resolved.

\section{SFA-FRAP Instrumentation}

The primary instrumentation of conventional SFA-FRAP consists of a laser source, a dichroic mirror, two fast shutters, a galvanometer-driven movable mirror, an objective lens, a CCD camera and mercury arc lamp, and an image recording system such as a frame grabber card (Fig. 4). The laser source is directed through one shutter and dichroic mirror to the objective lens and into the fluorescent sample, whereas the lamp is directed by the movable mirror to be collinear with the laser.

The laser is typically an argon-ion laser as in FRAP. SFA-FRAP is traditionally used in thick (many hundreds of micrometers) tissues, and the imaged bleach spot is several tens of micrometers wide. Consequently, the diffusive recovery times can be relatively slow (several tens of milliseconds to many minutes), and the laser modulation rate does not have to be as rapid as in FRAP. Furthermore, the laser modulation is binary, with a bright bleaching flash and zero power being the only required states, and no intermediate power monitoring beam is needed. Therefore, a fast shutter is generally sufficient for SFA-FRAP, instead of a rapid (and more expensive) analog device such as the AOM or Pockels cell.

\section{SFA-FRAP Procedure}

1. Shutter the laser so that the movable mirror directs the mercury lamp illumination into the sample. The CCD records a few prebleach images.

2. Shutter the CCD to avoid damage, and move the movable mirror out of the laser path. Open the laser shutter, allowing the bleaching flash through. Close the laser shutter again, ending the bleaching flash, and unshutter the CCD.

3. The movable mirror periodically shifts back into the light path, directing the mercury arc lamp illumination into the objective lens and allowing epifluorescence images to be captured as the bleached distribution recovers to equilibrium levels and the bleached spot disappears.

4. The series of images of the evolution of the bleached spot are Fourier transformed, and six of the lowest spatial frequencies are plotted. The exponential decay of the spatial frequencies yields the diffusion coefficient of the labeled molecule as well as the fraction of immobile fluorophores.

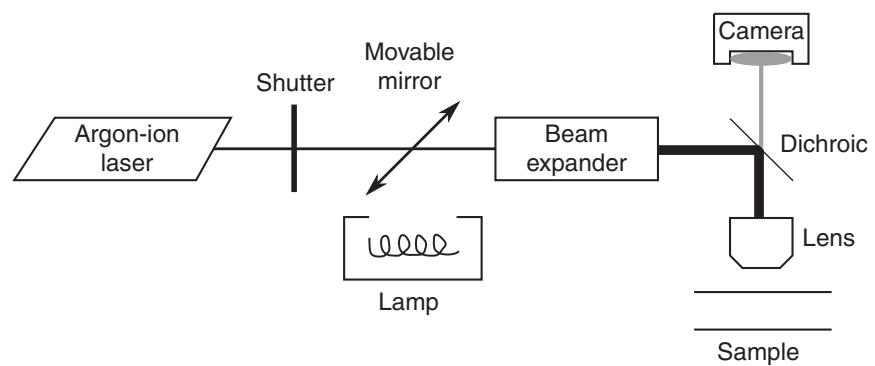

FIGURE 4. Equipment for FRAP with spatial Fourier analysis. Laser and lamp light are passed collinearly, but independently, through the beam expander and are directed by the dichroic to the objective lens, which focuses the light within the sample. The presence of the laser light is modulated by the shutter, whereas the movable mirror controls the presence of lamp light. Fluorescence from the sample is passed back through the lens and the dichroic to the CCD camera. 
K.D. Sullivan et al.

\section{Considerations when Determining SFA-FRAP Diffusion Parameters}

A number of steps must be taken to ensure accurate determination of the diffusion parameters.

1. The epifluorescence lamp must be bright enough to generate a sufficient signal, but not so bright as to cause significant photobleaching. Photobleaching caused by the lamp can be easily detected by performing a test SFA-FRAP experiment with no bleach pulse. If the fluorescence signal decays significantly over the course of the image series, the epifluorescence lamp must be attenuated. Additionally, the duty cycle of the monitoring pulses can be altered, thereby reducing the total exposure time of the sample during the recovery period.

2. The duration of the bleaching flash must be short enough that no significant diffusion (i.e., recovery in fluorescent signal) occurs during the bleach pulse. A rule of thumb is that the bleach pulse must be less than one-tenth of the half-recovery time of the subsequent spatial frequency decay curve.

3. If the acquisition of the fluorescence recovery images does not occur for a long enough period, overestimation of the immobile fraction and underestimation of the diffusion recovery time can result. A rule of thumb is that the decay curve of the spatial frequencies (see below) should be visibly flat (any change is less than shot noise) for the latter half of the recording time.

Diffusion Analysis

After a photobleaching pulse, the concentration distribution of unbleached fluorophores $c(x, y, t)$ evolves according to the diffusion equation. If the concentration distribution is first Fourier transformed with respect to $x$ and $y$, the solution to this differential equation is a simple exponential (Berk et al. 1993),

$$
C(u, v, t)=C(u, v, 0) e^{-\pi^{2}\left(u^{2}+v^{2}\right) D t},
$$

where $u$ and $v$ are the spatial frequencies and $D$ is the diffusion coefficient. The relationship between dye concentration at the sample and intensity at the corresponding location in the CCD image may vary in space but is expected to be constant in time. Therefore, the Fourier transform of the CCD images also decays with a simple exponential in which the exponential decay time of a given pair of spatial frequencies is $1 /\left(4 \pi^{2}\left(u^{2}+v^{2}\right) D\right)$. To analyze SFA-FRAP data, the series of CCD images of the evolving dye distribution are each Fourier transformed, and the exponential decay of a number (typically six) of selected spatial frequency pairs is analyzed to produce the exponential decay time, which directly yields the diffusion coefficient. Unlike FRAP or MPFRAP, no knowledge of the initial spatial distribution of photobleaching is required. Note that this analysis ignores diffusion along the optical axis and, furthermore, uses epifluorescence images of the diffusing system, therefore generating a diffusion coefficient that is an average over the visible depth of the system. In other words, any depth-dependent differences in the diffusion coefficient will be averaged out to a single value (Berk et al. 1993).

Single-photon and multiphoton FRAP can also be used to measure the diffusional coupling between two connected compartments. A characteristic time course for the diffusion or the transport of different fluorescent molecules can be obtained by bleaching one compartment and by monitoring the fluorescence recovery curve as it is refilled with a fluorophore from the unbleached compartment. This information can then be used to determine parameters such as resistivity and pore size of the separating barrier. MPFRAP has been used to examine diffusion of dyes between the excitatory synapse (dendritic spine) and its parent dendrite (Svoboda et al. 1996; Majewska et al. 2000; Sobczyk et al. 2005) and between plant plastids (Kohler et al. 1997). FRAP has examined the cell 
cytoplasm and the nucleus (Wei et al. 2003) as well as the turnover of fluorescently tagged actin filaments between the spine and the dendrite (Star et al. 2002).

\section{Instrumentation for Compartmentalization Analysis}

A FRAP or MPFRAP instrument (described above) can be used for compartmentalization analysis. Because diffusion between compartments tends to be slower than diffusion within a compartment, these experiments can often be performed in the line-scan mode on a laser-scanning microscope. In the line-scan mode, the excitation beam is scanned repeatedly along a single line that intersects an object of interest, and a position-versus-time curve of the fluorescence is generated. The line-scan mode uses the acquisition electronics of the laser-scanning microscope and obviates the need to purchase a separate photon-counting device. This mode can limit the acquisition speed, however, depending on the design of the microscope.

\section{Compartmentalization Analysis Procedure}

1. Choose one of the compartments for bleaching and monitoring (typically the smaller compartment). Set the laser modulator (AOM or Pockels cell) to a low transmission state, producing the monitor beam and generating fluorescence from a region of interest (ROI) within the sample, which is collected by the objective lens and is detected by the PMT as a measure of the prebleach fluorescence.

2. Gate the PMT (i.e., the dynode voltage is set to 0 ) to avoid damage, and switch the laser modulator to a high transmission level, producing the bleach beam, which photobleaches a fraction of the fluorophores within the ROI. The modulator rapidly returns to the low monitor state (after a total bleach time that depends on the sample dynamics, as described above), and the PMT is subsequently ungated.

3. Continuously record the fluorescence generated in the ROI by the monitor beam. As unbleached fluorophores diffuse in from the unbleached compartment, the fluorescence signal recovers back to equilibrium levels.

4. Analyze the fluorescence recovery curve to yield the characteristic coupling time as well as the fraction of immobile fluorophores.

\section{Considerations when Determining Compartmentalization}

Analysis Diffusion Parameters

1. Bleaching during the monitoring phase, the bleach duration, and the total acquisition time must be evaluated as in points $1-3$ in the section, Considerations when Determining FRAP and MPFRAP Diffusion Parameters, described above.

2. Diffusional coupling is typically studied between two well-mixed compartments (i.e., compartments in which the timescale of diffusional equilibrium within the compartments is much faster than between compartments). This can be verified by spot bleaching within each of the compartments to determine the diffusion characteristics for fluorophores in each of the compartments.

3. Because the communicating compartments are well mixed, the initial spatial distribution of bleached molecules is irrelevant because the bleached compartment undergoes diffusive mixing before significant communication with other compartments can occur. Consequently, neither the bleach spot profile nor the excitation saturation is a significant concern. However, it is important to determine that bleaching occurs in only one compartment. This can be accomplished by performing a line scan that intersects both compartments while restricting the bleaching pulse to a single compartment. In this case, both compartments can be monitored to ensure that bleaching is spatially restricted. 
K.D. Sullivan et al.

\section{Compartmentalization Analysis}

The fluorescence recovery curve in a well-mixed photobleached compartment diffusionally coupled to larger well-mixed compartments is given by (Svoboda et al. 1996; Majewska et al. 2000)

$$
F(t)=F(\infty)-\Delta F_{0} e^{-t / \tau},
$$

where $F(\infty)$ is the fluorescence at $t=\infty, \Delta F_{0}$ is the change in fluorescence level following the bleach pulse, and $\tau$ is the timescale of diffusion between the two compartments. The timescale $\tau$ of recovery between compartments provides insight into the characteristic resistivity of the coupling pathway, the number of coupling pathways, the diffusion coefficient of the tracer, etc., depending on the geometry of the system (Majewska et al. 2000; Wei et al. 2003).

\section{SUMMARY}

FRAP is a valuable tool for measuring the diffusion coefficient (or analogous transport parameters) in biological samples. The three FRAP techniques described here vary in resolution and depth penetration. FRAP requires thin samples $(\sim 1 \mu \mathrm{m})$ and yields an effective two-dimensional diffusion coefficient. SFA-FRAP is not limited by sample size but offers a diffusion coefficient averaged over the axial extent of the light cone of the objective. In addition, MPFRAP offers three-dimensional resolution and greatly improved depth penetration. Another common technique for measuring transport, fluorescence correlation spectroscopy (FCS), also comes in one-photon and two-photon varieties (Madge et al. 1974; Berland et al. 1995) and offers analogous resolution and depth penetration to the FRAP family. FRAP and FCS form a strong complement because FCS requires low fluorophore concentrations and is highly sensitive to background noise, whereas FRAP requires high fluorophore concentrations and is, therefore, less sensitive to background noise.

The relevance of two-photon FRAP, in particular, appears to grow as biomedical research delves further in vivo. As described above, MPFRAP was recently expanded to allow for the accurate measurement of diffusion in the presence of convective flow, making it a strong candidate for use in leaky physiological systems. Because of its superior spatial confinement, MPFRAP may also prove effective at measuring diffusion in the presence of bounding geometries, allowing it to be taken inside cell compartments, for example. There is also great promise for MPFRAP to take the leap into clinical applications via endoscopy.

\section{REFERENCES}

Abney JR, Scalettar BA, Thompson NL. 1992. Evanescent interference patterns for fluorescence microscopy. Biophys J 61: 542-552.

Axelrod D, Koppel DE, Schlessinger J, Elson E, Webb WW. 1976. Mobility measurement by analysis of fluorescence photobleaching recovery kinetics. Biophys J 16: 1055-1069.

Berk DA, Yuan F, Leunig M, Jain RK. 1993. Fluorescence photobleaching with spatial Fourier analysis: Measurement of diffusion in light-scattering media. Biophys J 65: 2428-2436.

Berk DA, Yuan F, Leunig M, Jain RK. 1997. Direct in vivo measurement of targeted binding in a human tumor xenograft. Proc Natl Acad Sci 94: 1785-1790.

Berland KM, So PT, Gratton E. 1995. Two-photon fluorescence correlation spectroscopy: Method and application to the intracellular environment. Biophys J 68: 694-701.

Braeckmans K, Remaut K, Vandenbroucke RE, Lucas B, De Smedt SC, Demeester J. 2007. Line FRAP with the confocal laser scanning microscope for diffusion measurements in small regions of 3-D samples. Biophys J 92: 2172-2183.

Braga J, McNally JG, Carmo-Fonseca M. 2007. A reaction-diffusion model to study RNA motion by quantitative fluorescence recovery after photobleaching. Biophys J 92: 2694-2703.
Brown EB, Wu ES, Zipfel W, Webb WW. 1999. Measurement of molecular diffusion in solution by multiphoton fluorescence photobleaching recovery. Biophys J 77: 2837-2849.

Cardarelli F, Serresi M, Bizzarri R, Giacca M, Beltram F. 2007. In vivo study of HIV-1 Tat arginine-rich motif unveils its transport properties. Mol Ther 15: 1313-1322.

Chary SR, Jain RK. 1989. Direct measurement of interstitial convection and diffusion of albumin in normal and neoplastic tissues by fluorescence photobleaching. Proc Natl Acad Sci 86: 5385-5389.

Chauhan VP, Lanning RM, Diop-Frimpong B, Mok W, Brown EB, Padera TP, Boucher Y, Jain RK. 2009. Multiscale measurements distinguish cellular and interstitial hindrances to diffusion in vivo. Biophys J 97: 330-336.

Denk W, Strickler JH, Webb WW. 1990. Two-photon laser scanning microscopy. Science 248: 73-76.

Feder TJ, Brust-Mascher I, Slattery JP, Baird B, Webb WW. 1996. Constrained diffusion or immobile fraction on cell surfaces: A new interpretation. Biophys J 70: 2767-2773.

Kaufmann EN, Jain RK. 1991. Measurement of mass transport and reaction parameters in bulk solution using photobleaching. Reaction limited binding regime. Biophys J 60: 596-610. 
Kohler RH, Cao J, Zipfel WR, Webb WW, Hanson MR. 1997. Exchange of protein molecules through connections between higher plant plastids. Science 276: 2039-2042.

Li J, Sullivan KD, Brown EB, Anthamatten M. 2010. Thermally activated diffusion in reversibly associating polymers. Soft Matter 6: 235-238.

Madge D, Elson EL, Webb WW. 1974. Fluorescence correlation spectroscopy. II. An experimental realization. Biopolymers 13: 29-61.

Majewska A, Brown E, Ross J, Yuste R. 2000. Mechanisms of calcium decay kinetics in hippocampal spines: Role of spine calcium pumps and calcium diffusion through the spine neck in biochemical compartmentalization. J Neurosci 20: 1722-1734.

Mazza D, Braeckmans K, Cella F, Testa I, Vercauteren D, Demeester J, De Smedt SS, Diaspro A. 2008. A new FRAP/FRAPa method for threedimensional diffusion measurements based on multiphoton excitation microscopy. Biophys J 95: 3457-3469.

Pawley J, ed. 2006. Handbook of biological confocal microscopy Springer, New York.

Peters R, Peters J, Tews K, Bahr W. 1974. Microfluorimetric study of translational diffusion of proteins in erythrocyte membranes. Biochim Biophys Acta 367: 282-294.

Pluen A, Boucher Y, Ramanujan S, McKee TD, Gohongi T, di Tomaso E, Brown EB, Izumi Y, Campbell RB, Berk DA, et al. 2001. Role of tumorhost interactions in interstitial diffusion of macromolecules: Cranial vs. subcutaneous tumors. Proc Natl Acad Sci 98: 4628-4633.

Schneider MB, Webb WW. 1981. Measurement of submicron laser beam radii. Appl Opt 20: 1382-1388.

Schulmeister S, Ruttorf M, Thiem S, Kentner D, Lebiedz D, Sourjik V. 2008. Protein exchange dynamics at chemoreceptor clusters in Escherichia coli. Proc Natl Acad Sci 105: 6403-6408.
Sobczyk A, Scheuss V, Svoboda K. 2005. NMDA receptor subunit-dependent $\left[\mathrm{Ca}^{2+}\right]$ signaling in individual hippocampal dendritic spines. $J$ Neurosci 25: 6037-6046.

Star EN, Kwiatkowski DJ, Murthy VN. 2002. Rapid turnover of actin in dendritic spines and its regulation by activity. Nat Neurosci 5: 239-246.

Stroh M, Zipfel WR, Williams RM, Ma SC, Webb WW, Saltzman WM. 2004. Multiphoton microscopy guides neurotrophin modification with poly (ethylene glycol) to enhance interstitial diffusion. Nat Mater 3: 489494.

Sullivan KD, Sipprell WH III, Brown EB Jr, Brown EB III. 2009. Improved model of fluorescence recovery expands the application of multiphoton fluorescence recovery after photobleaching in vivo. Biophys $J$ 96: 50825094.

Svoboda K, Tank DW, Denk W. 1996. Direct measurement of coupling between dendritic spines and shafts. Science 272: 716-719.

Tsay TT, Jacobson KA. 1991. Spatial Fourier analysis of video photobleaching measurements. Principles and optimization. Biophys J 60: 360-368.

Waharte F, Brown CM, Coscoy S, Coudrier E, Amblard F. 2005. A twophoton FRAP analysis of the cytoskeleton dynamics in the microvilli of intestinal cells. Biophys J 88: 1467-1478.

Wedekind P, Kubitscheck U, Heinrich O, Peters R. 1996. Line-scanning microphotolysis for diffraction-limited measurements of lateral diffusion. Biophys J 71: 1621-1632.

Wei X, Henke VG, Strubing C, Brown EB, Clapham DE. 2003. Real-time imaging of nuclear permeation by EGFP in single intact cells. Biophys J 84: 1317-1327.

Zipfel WR, Williams RM, Christie R, Nikitin AY, Hyman BT, Webb WW. 2003. Live tissue intrinsic emission microscopy using multiphotonexcited native fluorescence and second harmonic generation. Proc Natl Acad Sci 100: 7075-7080. 


\section{Single- and Two-Photon Fluorescence Recovery after Photobleaching}

Kelley D. Sullivan, Ania K. Majewska and Edward B. Brown

Cold Spring Harb Protoc; doi: 10.1101/pdb.top083519

\begin{tabular}{|c|c|}
\hline $\begin{array}{l}\text { Email Alerting } \\
\text { Service }\end{array}$ & Receive free email alerts when new articles cite this article - click here. \\
\hline $\begin{array}{l}\text { Subject } \\
\text { Categories }\end{array}$ & $\begin{array}{l}\text { Browse articles on similar topics from Cold Spring Harbor Protocols. } \\
\text { Cell Biology, general (1382 articles) } \\
\text { Cell Imaging (525 articles) } \\
\text { Fluorescence ( } 517 \text { articles) } \\
\text { Fluorescence, general ( } 341 \text { articles) } \\
\text { Imaging/Microscopy, general (579 articles) } \\
\text { Multi-Photon Microscopy (103 articles) } \\
\text { Photobleaching (12 articles) }\end{array}$ \\
\hline
\end{tabular}

